\title{
Biomedical Semantics: the Hub for Biomedical Research 2.0
}

\author{
Dietrich Rebholz-Schuhmann* and Goran Nenadic
}

\author{
* Correspondence: \\ rebholz@ebi.ac.uk \\ 1 European Bioinformatics \\ Institute, Wellcome Trust \\ Genome Campus, Hinxton, \\ Cambridge, CB10 1SD, UK \\ Full list of author information is \\ available at the end of the article
}

We would like to welcome you to Journal of Biomedical Semantics (JBMS), a new openaccess, peer-reviewed inter-disciplinary journal that addresses novel theoretical and practical advances in biomedical semantics research. The main focus of JBMS is the development and integration of semantics resources into the biomedical research practice. The journal therefore aims at providing a forum for dissemination of achievements that have generated or used semantics resources to tackle challenges in biology and medicine, for example in genetics, medical treatments, development of therapeutics, translational medicine, food production and others.

\section{The Rise of Biomedical Semantics}

New discoveries in biology and medicine over the last two decades have been facilitated by large-scale data generation exercises as part of high-throughput experiments [1]. The observed data is maintained in ever growing scientific public databases as structured data, while novel scientific findings are reported in a semi- or un-structured form in the literature. Many of the past and present developments in biomedicine, in particular in computational biology and medical informatics, have focused on structured data management. At the same time, there has been a remarkable increase in the number and coverage of semantics resources and semantically annotated repositories to leverage biomedical knowledge from their structured and unstructured origins alike [2].

As part of the new developments, biomedical research is not relying only on local data and findings generated within individual laboratories, but is increasingly integrating shared, publicly available biomedical repositories. Furthermore, researchers exploit data resources from miscellaneous domains: for example, molecular biologists using toxicological data and medical scientists exploring data resources from molecular biology or environmental sciences. Altogether, biomedical research is moving beyond approaches utilizing "handcrafted" hypotheses and inference techniques towards approaches that are trained on largescale, semantically integrated biomedical data. For example, research in systems biology and systems medicine requires semantic integration of data across the biomedical domain to feed it into comprehensive and formalised models of living beings, utilising techniques from computer science, mathematics and engineering to analyse and predict biological and medical outcomes based on experimental and formal semantics descriptions.

Indeed, in recent years, we have been witnessing an evolution towards formalising biomedical knowledge in explicit domain models such as curated and annotated datasets, ontologies, pathways, semantic networks, etc [3]. The biomedical semantics resourceome 
now contains a large number of repositories. Biomedical ontologies [4], terminologies and controlled vocabularies (e.g. the Gene Ontology [5], MeSH [6], UMLS [7], ICD-10 [8], etc.), in particular, have been widely used for the integration of diverse scientific databases and for standardisation of information access through common but stringent logical definitions and constraints on semantic annotations of database content. Such semantic descriptors have been also used for detailed analyses and improvement of our understanding of known and prediction of yet un-explained biomedical processes.

In addition to the development of the semantics resourceome for structured data, researchers have been targeting the automated integration and exploitation of unstructured data, i.e. the scientific literature, to capture novel results and findings [9]. Information retrieved from semantics-driven literature mining needs to be integrated into existing knowledge repositories, thus becoming an integral part of a fully specified and interconnected knowledge space for biomedical research.

The need for formalised semantics is, of course, not specific to biomedicine. However, the unprecedented scale of biomedical data has put biomedical research into a forefront position to foster the development of leading-edge technologies such as linked data and the Semantic Web. We can already see a growing penetration of these technologies into the life science community, evident through many initiatives and conferences (e.g. Bio2RDF [10], BioHackathon [11], SWAT4LS [12]). In addition to institutionally-supported actions, there are various community-driven initiatives to annotate, organise and provide access to the whole spectrum of biomedical resources, from primary experimental data to data analysis workflows (e.g. myExperiment [13]) and secondary semanticallycurated resources (e.g. ORegAnno [14]).

Management and maintenance of the semantics resourceome has already been recognised as one of the most challenging tasks that the community needs to address in order to facilitate our understanding of biological processes and predictions of functional behaviour. An interoperable and comprehensive semantic infrastructure is likely to facilitate data, knowledge and methodology re-use and integration, and thus encourage further developments in the field. Coupled with human- and machine-readable formal models and automated reasoning, the infrastructure can provide an environment for the next generation of biomedical research, one that will rely on a distributed semantic grid of biomedical data and knowledge. Some authors already refer to these developments as Semantic Systems Biology (and Medicine), where reasoning and conclusions are based on the full integration of biomedical knowledge [15]. Combined with semantics-driven data analysis workflow orchestration and distributed execution, the new framework for in-silico biomedical experimentation has the potential to add a new dimension to the way biomedical research is conducted [16].

One question that many biomedical semantics researchers often face is whether their research aims to create a virtual scientist, who would do automated investigations and will, eventually, replace real-world scientists. While such attempts have been explored [17], most of the current research in the domain of biomedical semantics is focused on the facilitation of large-scale, systematic and formalised representation and exploration of biomedical knowledge, where the main onus is still on an expert to mange and experience the scientific discovery process, but using a research environment that can cope with the complexity, dynamics and volume of biomedical knowledge. 
Biomedical semantics research thus aims to bridge the gap between the data and knowledge that has been increasingly available and facilitate their use to support scientific exploration. Only full semantic integration of biomedical knowledge and experimental data can provide a means for scientists to model complex biological systems and thus improve our understanding of living organisms.

\section{JBMS: towards the next generation of biomedical investigations}

JBMS is primarily focused on the engineering, population and scientific exploitation of semantic resources in biomedicine. It encompasses the full variety of biomedical semantic resourceome (i.e. ontologies, taxonomies, terminologies, controlled vocabularies, annotated data, knowledge and service repositories, literature, reasoning systems, etc.) and their use in data and knowledge integration, mining, modelling, interpretation and exploitation in and for biomedical research. Examples of targeted scientific achievements include annotation and curation work of biomedical data resources, development of ontologies, inference of knowledge from publicly available data resources (e.g. exploiting data and text mining or reasoning techniques), and integration and exploitation of resources as part of the emerging Semantic Web solutions.

JBMS aims at publishing interdisciplinary research that crosses the traditional boundaries between the involved disciplines (i.e. biology, medicine, mathematics, computer science, bioinformatics) and areas (knowledge representation, information retrieval, data mining, reasoning and visualisation). The journal will therefore aim to attract contributions that are currently scattered over journals in different fields, and are typically focused on the perspective of a particular discipline (where the publication will be deposited), rarely addressing the entire complexity of biomedical semantic modelling and usage. The full scope of topics of interest is given on the journal's About page. The two main topic areas of the journal are:

(1) Infrastructure for biomedical semantics

This area focuses on development of semantic resources and the underlying infrastructure, including data and knowledge representation models; provenance, curation, evolution, validation, evaluation and dissemination of semantic resources; semantic integration and mapping between data resources, biomedical Semantic Web, etc.

(2) Exploitation of semantic resources

Typical examples would include using biomedical text and data mining for automated hypothesis generation; exploitation of the Semantic Web and linked data in biomedical research; large-scale automated analyses of biomedical data using ontology-driven reasoning for prediction of novel findings, etc.

JBMS aims to be in the forefront of scientific semantic publishing, and will work closely with both the publisher (BioMed Central) and research community to establish an infrastructure and best practice guidelines for developing and annotating semantic resources, including the publications themselves. We aspire to provide the authors and readers with the opportunities to semantically enrich the publications so that they could become part of an integrated semantics network of biomedicine.

We are very pleased to introduce the distinguished members of the Editorial Board. All members have significantly contributed to the research domains relevant to JBMS and will help to shape the development of the journal, providing both strategic guidelines and management of the peer-review process. The scientific interests of the Board members represent the broad range of topics that are relevant to JBMS, ranging from biology 
and medicine to bioinformatics, medical and health informatics, computer science and philosophy. We would like to express our gratitude to all of them for their engagement and help to establish and launch this journal.

The authors of the inaugural issue deserve our thanks and congratulations. The first manuscripts accepted for publication include contributions from the conference Languages in Biology and Medicine 2009 [18]. A special issue on biomedical ontologies will follow, featuring extended versions of the publications from the Bio-Ontology Special Interest Group meeting in 2009 [19].

We are also grateful to BioMed Central publishing company and its members of staff for the technical support and their encouragements to set up the journal, but also for leading the way in promoting the ideas and establishing research forums that cover emerging areas in biomedical sciences.

Altogether, we hope you will enjoy learning about the findings, challenges and solutions reported in JBMS, and that you will actively contribute to establishing biomedical semantics research as a key, next generation biomedical research area. We expect that the JBMS will become a hub for the dissemination of this type of research to the wider scientific community. We would therefore encourage all potential readers and authors to contribute their research work to JBMS and become part of this exciting research domain.

\section{Dietrich Rebholz-Schuhmann and Goran Nenadic \\ Editors-in-Chief}

\section{Author Details}

European Bioinformatics Institute, Wellcome Trust Genome Campus, Hinxton, Cambridge, CB10 1SD, UK

Received: 25 March 2010 Accepted: 31 March 2010

Published: 31 March 2010

\section{References}

1. Collins FS, Morgan M, Aristides P: The Human Genome Project: Lessons from Large-Scale Biology. Science 2003, 300:286-290.

2. Smith B, Ashburner M, Rosse C, Bard J, Bug W, Ceusters W, Goldberg LJ, Eilbeck K, Ireland A, Mungall CJ, OBI Consortium, Leontis N, Rocca-Serra P, Ruttenberg A, Sansone SA, Scheuermann RH, Shah N, Whetzel PL, Lewis S: The OBO Foundry: coordinated evolution of ontologies to support biomedical data integration. Nat Biotechno/ 2007, 25(11):1251-5.

3. Noy NF, Shah NH, Whetzel PL, Dai B, Dorf M, Griffith N, Jonquet C, Rubin DL, Storey MA, Chute CG, Musen MA: BioPortal: ontologies and integrated data resources at the click of a mouse. Nucleic Acids Res 2009:W170-3.

4. The Open Biological and Biomedical Ontologies [http://www.obofoundry.org/]

5. Gene Ontology [http://www.geneontology.org]

6. Medical Subject Headings [http://www.ncbi.nlm.nih.gov/mesh

7. Unified Medical Language System [http://www.nlm.nih.gov/research/umls/]

8. International Classification of Diseases [http://www.who.int/classifications/icd/en/

9. van Haagen HH, 't Hoen PA, Botelho Bovo A, de Morrée A, van Mulligen EM, Chichester C, Kors JA, den Dunnen JT, van Ommen GJ, Maarel SM van der, Kern VM, Mons B, Schuemie MJ: Novel protein-protein interactions inferred from literature context. PLoS One 2009, 4(11):e7894.

10. Semantic Web atlas of postgenomic knowledge [http://bio2rdf.org/]

11. BioHackathon 2010 [http://hackathon3.dbcls.jp/]

12. Semantic Web Applications and Tools for Life Sciences [http://www.swat4ls.org/]

13. De Roure D, Goble C, Stevens R: The Design and Realisation of the myExperiment Virtual Research Environment for Social Sharing of Workflows. Future Generation Computer Systems 2009, 25:561-567. doi:10.1016/ j.future.2008.06.010

14. Griffith OL, Montgomery SB, Bernier B, Chu B, Kasaian K, Aerts S, Mahony S, Sleumer MC, Bilenky M, Haeussler M, Griffith M, Gallo SM, Giardine B, Hooghe B, Van Loo P, Blanco E, Ticoll A, Lithwick S, Portales-Casamar E, Donaldson IJ, Robertson AG, Wadelius C, De Bleser P, Vlieghe D, Halfon MS, Wasserman WW, Hardison R, Bergman CM, Jones SJM, the Open Regulatory Annotation Consortium: "ORegAnno: an open-access community-driven resource for regulatory annotation". Nucleic Acids Research 2008.

15. Antezana E, Blonde W, Egana M, Rutherford A, Stevens R, De Baets B, Mironov V, Kuiper M: Structuring the life science resourceome for Semantic Systems Biology: lessons from the BioGateway project. In SWAT4LS-Semantic Web Applications and Tools for Life Sciences e-Science Institute, Edinburgh, Scotland, UK; 2008. 
16. Bourne PE, Beran B, Bi C, Bluhm W, Dunbrack R, et al: Will Widgets and Semantic Tagging Change Computational Biology? PLoS Comput Bio/ 2010, 6(2):e1000673. doi:10.1371/journal.pcbi.1000673

17. King RD, Whelan KE, Jones FM, Reiser PG, Bryant CH, Muggleton SH, Kell DB, Oliver SG: Functional genomic hypothesis generation and experimentation by a robot scientist. Nature 2004, 427(6971):247-52.

18. Rebholz-Schuhmann D, Collier N, Park JC, Wong LS: Wrestling with biomedical research results: Language resources and literature analysis. J Bioinform Comput Biol 2010, 8(1):129-130.

19. Bio-Ontologies 2009: Knowledge in Biology [http://bio-ontologies.org.uk/2009/]

doi: $10.1186 / 2041-1480-1-1$

Cite this article as: Rebholz-Schuhmann and Nenadic, Biomedical Semantics: the Hub for Biomedical Research 2.0 Journal of Biomedical Semantics 2010, 1:1

Submit your next manuscript to BioMed Central and take full advantage of:

- Convenient online submission

- Thorough peer review

- No space constraints or color figure charges

- Immediate publication on acceptance

- Inclusion in PubMed, CAS, Scopus and Google Scholar

- Research which is freely available for redistribution 